\title{
Identifying Corticothalamic Network Epicenters in Patients with Idiopathic Generalized Epilepsy
}

\author{
G.-J. Ji, Z. Zhang, Q. Xu, Z. Wang, J. Wang, Q. Jiao, F. Yang, Q. Tan, G. Chen, Y.-F. Zang, W. Liao, and G. Lu
}

\begin{abstract}
BACKGROUND AND PURPOSE: Corticothalamic networks are considered core pathologic substrates for idiopathic generalized epilepsy; however, the predominant epileptogenic epicenters within these networks are still largely unknown. The current study aims to identify these epicenters by resting-state functional connectivity.
\end{abstract}

MATERIALS AND METHODS: To identify epicenters within the corticothalamic networks in idiopathic generalized epilepsy, we retrospectively studied a large cohort of patients with this condition $(n=97)$ along with healthy controls $(n=123)$ by resting-state functional MR imaging. The thalamus was functionally divided into subregions corresponding to distinct cortical lobes for 5 parallel corticothalamic networks. The functional connectivity between each voxel in the cortical lobe and the corresponding thalamic subregion was calculated, and functional connectivity strength was used to evaluate the interconnectivity of voxels in the cortex and thalamus.

RESULTS: The projection of 5 cortical lobes to the thalamus is consistent with previous histologic findings in humans. Compared with controls, patients with idiopathic generalized epilepsy showed increased functional connectivity strength in 4 corticothalamic networks: 1) the supplementary motor area, pulvinar, and ventral anterior nucleus in the prefrontal-thalamic network; 2) the premotor cortex and ventrolateral nucleus in motor/premotor-thalamic networks; 3) the visual cortex, posterior default mode regions, and pulvinar in parietal/ occipital-thalamic networks; and 4) the middle temporal gyrus in the temporal-thalamic network.

CONCLUSIONS: Several key nodes were distinguished in 4 corticothalamic networks. The identification of these epicenters refines the corticothalamic network theory and provides insight into the pathophysiology of idiopathic generalized epilepsy.

ABBREVIATIONS: FCS = functional connectivity strength; GSWD = generalized spike-wave discharge; IGE = idiopathic generalized epilepsy; SMA = supplementary motor area

diopathic generalized epilepsy (IGE) is a common subtype of epilepsy involving abnormally synchronized generalized spikewave discharges (GSWDs) rapidly propagating to distributed networks. ${ }^{1}$ Various theories have been proposed to explain the origin and mechanism of generalized seizures ${ }^{2}$; the corticothalamic net-

\footnotetext{
Received August 28, 2014; accepted after revision January 17, 2015.

From the Center for Cognition and Brain Disorders and the Affiliated Hospital (G.J.J., J.W., Y.-F.Z., W.L.), Hangzhou Normal University, Hangzhou, China; Zhejiang Key Laboratory for Research in Assessment of Cognitive Impairments (G.-J.., J.W., Y.-F.Z., W.L.), Hangzhou, China; Departments of Medical Imaging (Z.Z., Q.X., W.L., G.L.), Neurology (F.Y., G.C.), and Neurosurgery (Q.T.), Jinling Hospital, Nanjing University School of Medicine, Nanjing, China; Department of Medical Imaging (Z.W.), Nanjing Drum Tower Hospital, the Affiliated Hospital of Nanjing University Medical School, Nanjing, China; and Department of Radiology (Q.J.), Taishan Medical University, Tai'an, China.

G.-J. Ji and Z. Zhang contributed equally to the article.

This research was supported by the Natural Science Foundation of China (grants 81401400, Gong-Jun Ji; 81401402, Qiang Xu; 81201155, 81471653, Wei Liao; 81301198 , Zhengge Wang; 81171328, Qing Jiao; 81201078, Fang Yang; 81271553, 81422022, Zhiqiang Zhang; 81020108022, 31471084, Yu-Feng Zang), China Postdoctoral Science Foundation (grant 2013M532229, Wei Liao), and Qian Jiang Distinguished Professor program (YuFeng Zang).
}

work has been suggested as a preferred target for the modification or elimination of seizure discharges. ${ }^{3,4}$

There is substantial evidence that dysfunction in the corticothalamic circuitry contributes to the pathogenesis of generalized seizures. ${ }^{3,4}$ Hemodynamic changes associated with GSWDs have been consistently observed in the thalamus and default mode areas. ${ }^{5}$ Recently, corticothalamic interactions in generalized epilepsy have been characterized by morphometric covariance, ${ }^{6}$ functional and anatomic connectivity, ${ }^{7,8}$ and causal influence. ${ }^{9}$ In particular, corticothalamic connections (parcellated through probabilistic tractography) showed abnormal functional connectivity in juvenile myoclonic epilepsy. ${ }^{7}$ However, little attention

\footnotetext{
Please address correspondence to Wei Liao, PhD, Center for Cognition and Brain Disorders and the Affiliated Hospital, Hangzhou Normal University, Hangzhou 310015, China; e-mail: weiliao.wl@gmail.com; and Guangming Lu, MD, Department of Medical Imaging, Nanjing Jinling Hospital, 305, Eastern Zhongshan Rd, Nanjing 210002, China; e-mail: cjr.luguangming@vip.163.com

- Indicates open access to non-subscribers at www.ajnr.org

http://dx.doi.org/10.3174/ajnr.A4308
} 
Table 1: Characteristics of patients and normal controls

\begin{tabular}{lcccc}
\hline \multicolumn{1}{c}{ Characteristic } & IGE $(\boldsymbol{n}=\mathbf{8 6})$ & NC $(\boldsymbol{n}=123)$ & $\boldsymbol{\chi}^{2} / \boldsymbol{t}$ Value & $\boldsymbol{P}$ Value \\
\hline Sex (male/female) & $31 / 55$ & $56 / 67$ & 1.87 & $.17^{\mathrm{a}}$ \\
Age (yr) & $25.77 \pm 6.31^{\mathrm{b}}$ & $25.49 \pm 7.15^{\mathrm{b}}$ & 1.22 & $.22^{\mathrm{c}}$ \\
Handedness (right/left) & $86 / 0$ & $123 / 0$ & - & - \\
Duration (yr) & $8.93 \pm 8.41^{\mathrm{b}}$ & - & - & - \\
Onset age (yr) & $16.84 \pm 7.93^{\mathrm{b}}$ & - & - & - \\
Frequency (times/yr) & $19.02 \pm 58.11^{\mathrm{b}}$ & - & - & - \\
\hline
\end{tabular}

Note:-NC, normal controls; -, not applicable.

${ }^{\mathrm{a}} \chi^{2}$ test.

${ }^{\mathrm{b}}$ Data represent mean $\pm \mathrm{SD}$.

'Two-sample $t$ test.

has been paid to the functional topographic pathways linking distinct cortical areas and specific thalamic nuclei. ${ }^{10,11}$ These projections likely have distinct roles in the mechanism of GSWDs, ${ }^{12}$ so it is essential to investigate corticothalamic functional networks in generalized epilepsy. More precisely, characterizing epicenters within each network may facilitate the development of targeted surgical interventions (eg, deep brain stimulation) that selectively disrupt seizures and ultimately improve the clinical treatment of IGE. $^{4}$

It was recently demonstrated that resting-state functional connectivity can reveal distinct corticothalamic networks ${ }^{13}$ analogous to classic histologic parcellation. ${ }^{10}$ As in previous investigations, ${ }^{14-16}$ a similar approach was adopted here to identify corticothalamic networks. We hypothesized that the functional synchronization of corticothalamic networks is altered in patients with IGE. To localize epicenters within these networks, we conducted a voxelwise comparison of network functional connectivity between patients and controls.

\section{MATERIALS AND METHODS \\ Participants}

Patients were consecutively enrolled at Jinling Hospital, Nanjing, China. Patients were diagnosed according to International League Against Epilepsy criteria (2001). The inclusion criteria for patients were as follows: 1) manifestation of typical clinical symptoms of idiopathic generalized tonic-clonic seizures, including tonic extension of the limbs, followed by a clonic phase of rhythmic jerking of the extremities, loss of consciousness during seizures without precursory symptoms of partial epilepsy and aura; 2) no evidence of secondary generalized seizures, such as trauma, tumor, intracranial infection; 3) no abnormality on structural MR imaging; 4) presence of GSWDs on the video-electroencephalogram; and 5) right-handedness. Ninety-seven patients fulfilled these inclusion criteria. The exclusion criteria were as follows: 1) history of addictions or neurologic diseases other than epilepsy; 2) history of partial seizures; 3) self-reported falling asleep during resting-state fMRI scanning; and 4) head translation or rotation parameters exceeding $\pm 1.5 \mathrm{~mm}$ or $\pm 1.5^{\circ}$. After excluding 11 patients due to excessive head motion, we included 86 patients in the final dataset (Table 1). All 86 patients had generalized tonic-clonic seizures, and 17 had additional myoclonic jerks and absences. All 86 patients were taking antiepileptic drugs, including valproic acid, topiramate, lamotrigine, phenobarbitone, or some combination, and 34 had taken other medications, including carbamazepine, phenytoin, traditional Chinese herbal medicines, oxcarbazepine, or clonazepam.
Healthy controls $(n=123)$ were recruited from the staff of Jinling Hospital. They had no history of neurologic disorders or psychiatric illnesses and no gross abnormalities in brain MRI.

Written informed consent was obtained from all participants. The study was approved by the local medical ethics committee at Jinling Hospital.

\section{Data Acquisition}

Data were acquired by using a Magnetom Trio (Siemens, Erlangen, Germany) MR imaging scanner at Jinling Hospital. Functional images were acquired during the interictal period. Scalp electroencephalograms were not recorded during scanning. All patients were assumed to be in the interictal state during scanning because there were no apparent seizure symptoms observed by video monitoring. Foam padding was used to minimize subject head motion. Functional images were acquired by using a single-shot, gradient-recalled echo-planar imaging sequence $\left(\mathrm{TR}=2000 \mathrm{~ms}, \mathrm{TE}=30 \mathrm{~ms}\right.$, flip angle $\left.=90^{\circ}\right)$. Thirty transverse sections $\left(\mathrm{FOV}=240 \times 240 \mathrm{~mm}^{2}\right.$, section thickness/intersection gap $=4 / 0.4 \mathrm{~mm}$ ) were acquired. A total of 250 volumes were acquired for each subject. Subjects were instructed to rest with their eyes closed, without thinking of anything in particular or falling asleep. After scanning, subjects were asked whether they had fallen asleep during the scan.

\section{Data Processing}

Preprocessing. Functional images were preprocessed by using the Data Processing Assistant for Resting-State fMRI (http:// rfmri.org/DPARSF) and SPM8 software (http://www.fil.ion. ucl.ac.uk/spm/software/spm8) toolkits. Functional images were section-timing-corrected, then registered to correct for head motion during the scan. On the basis of the known impact of head motion, the frame-wise displacement was calculated for each time point. ${ }^{17}$ If the frame-wise displacement exceeded $0.5 \mathrm{~mm}$, the value of the signal at that point was interpolated by using piecewise cubic Hermite. Functional images were normalized to the EPI template in Montreal Neurological Institute space and resampled to an isotropic $2-\mathrm{mm}^{3}$ voxel. Next, linear trends were removed and temporal bandpass filtering $(0.01-$ $0.08 \mathrm{~Hz}$ ) was performed. Finally, several sources of spurious variance were removed by regression of the following variables: 6 head-motion parameters, signals averaged over CSF, and white matter. ${ }^{13,14}$ As previously proposed, ${ }^{13}$ data were neither smoothed nor regressed out of the averaged global mean signal.

Identification of Functional Corticothalamic Networks. To identify functional corticothalamic networks, we divided bilateral hemispheres into 5 nonoverlapping cortical lobes based on anatomic templates ${ }^{13}:$ 1) prefrontal cortex, 2) motor/premotor cortex, 3) somatosensory cortex, 4) parietal/occipital cortex, and 5) temporal cortex. We calculated the functional connectivity between the averaged signal within each lobe and each voxel of the thalamus by using partial correlation for each subject. ${ }^{13}$ The thalamus mask was acquired from an automated anatomic labeling 


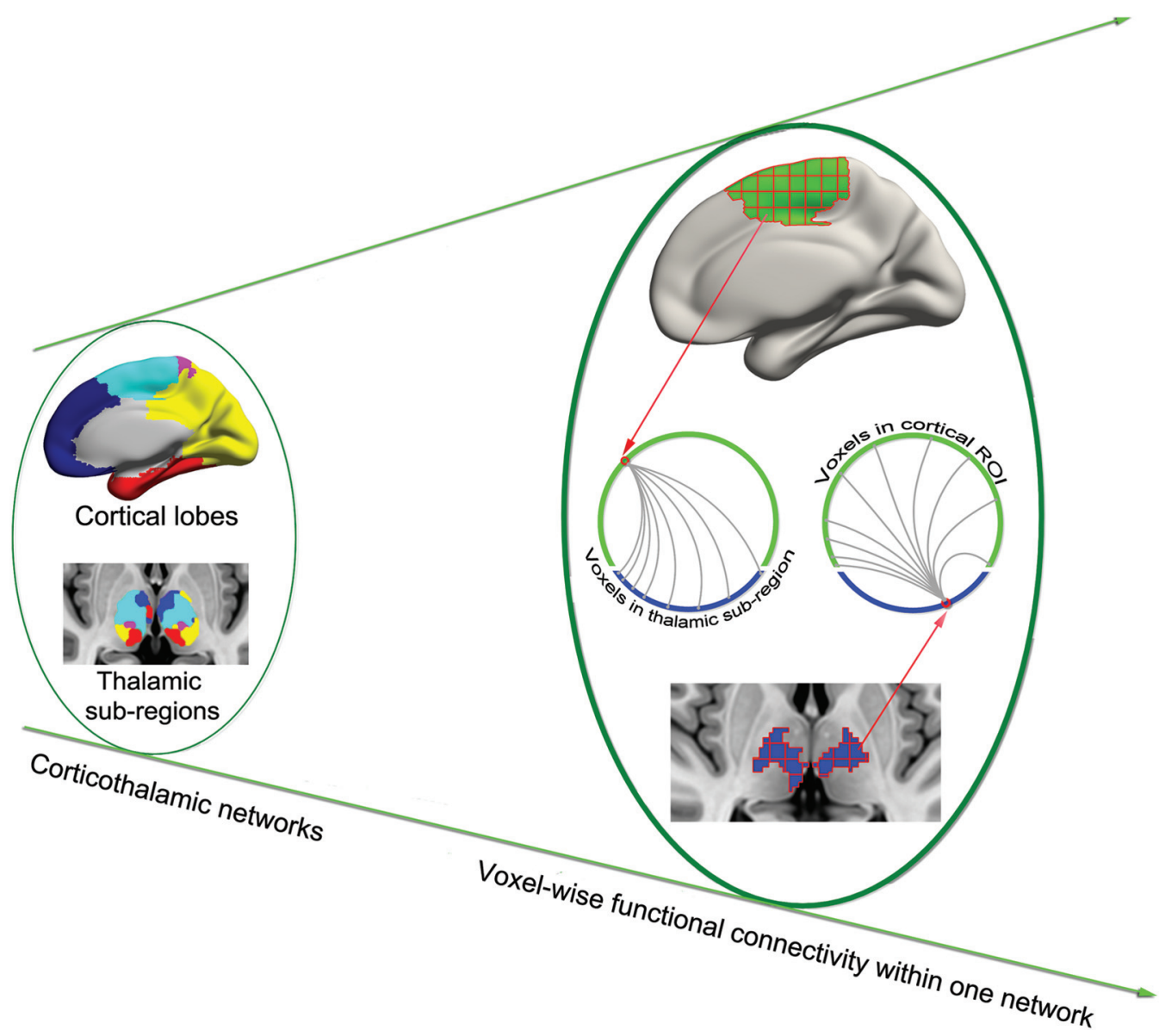

FIG 1. Schematic of data analysis. From the left to right planes, 5 corticothalamic networks were first identified; FCS was then calculated for each voxel within the corresponding corticothalamic network. For example, 1 voxel in the cortical lobe was correlated with all voxels in the corresponding thalamic subregion. All superthreshold coefficient values were averaged to obtain the FCS of the cortical voxel.

template. On the basis of previous studies, ${ }^{14}$ positive correlations were included in further analyses and transformed to $z$ scores by using the Fisher r-to-z transformation. $Z$ score maps were combined across subjects in each group by using a 1-sample $t$ test. Finally, each thalamic voxel was labeled according to the cortical lobe with the highest $t$ value (ie, "winner take all"). ${ }^{13-16}$ Thus, the thalamus was separately divided into 5 subregions in each group. To compute the functional connectivity strength (FCS) and compare this value between groups, we applied the winner-take-all approach to all participants $(n=209)$ to limit group bias during thalamic subregion creation. ${ }^{18}$

Voxelwise Functional Connectivity in Corticothalamic Networks. Because generalized epileptic seizures may originate at specific regions or epicenters rather than throughout the brain simultaneously, ${ }^{1}$ we sought to identify these epicenters in corticothalamic networks by using a voxelwise functional connectivity approach (Fig 1). ${ }^{19}$ Specifically, given $N$ voxels in a cortical lobe and $M$ voxels in the corresponding thalamic subregion, Pearson correlation analysis generated an $N \times M$ matrix for each participant-that is, for a given voxel in the cortical lobe, there were $M$ correlation values representing its functional synchronization with each voxel in the thalamic subregion. Only values above a predefined threshold (corresponding to $P=.05 / M$ ) were transformed to $z$ values and summed as the FCS value of cortical or thalamic subregion voxels. The FCS value is referred to as the "degree centrality" of weighted networks ${ }^{20}$; voxels with higher FCS values indicate a greater role in the heterogeneity of the capacity and intensity of connections. ${ }^{19}$

\section{Statistical Analysis}

FCS maps of 5 parallel corticothalamic networks were separately compared between groups by using the 2 -sample $t$ test. The corrected statistical threshold $(P<.05)$ was accomplished by 2 steps: First, the comparison result was corrected $(P<.01)$ by the AlphaSim program (http://afni.nimh.nih.gov/pub/dist/doc/ program_help/AlphaSim.html) within each network (with a height threshold of $P<.001$ for all cortical and thalamic subregions, and extent thresholds for each cortical region and the corresponding thalamic subregion of $>480$ and $24 \mathrm{~mm}^{3}$ [prefrontal cortex and thalamus], 160 and $32 \mathrm{~mm}^{3}$ [motor/premotor and thalamus], 720 and $24 \mathrm{~mm}^{3}$ [parietal/occipital cortex and thalamus], and 480 and $24 \mathrm{~mm}^{3}$ [temporal cortex and thalamus]). This step was followed by a Bonferroni correction for the 5 corticothalamic network comparisons. The clinical variables (disease duration, seizure frequency, and onset age) were correlated with regions showing abnormal FCS. Spearman correlation analysis was used because none of the clinical variables showed a Gaussian distribution. 


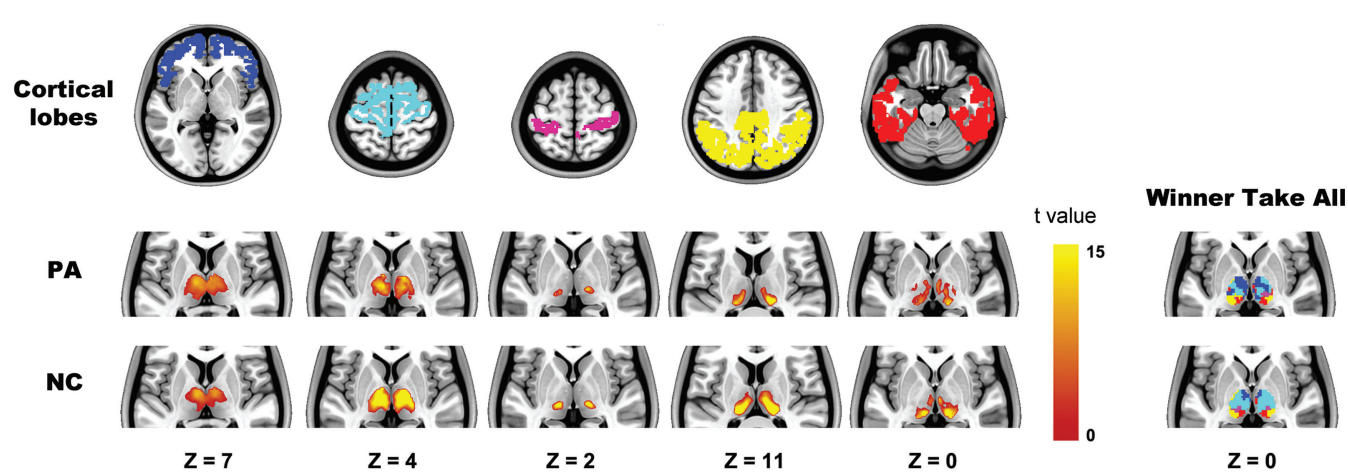

FIG 2. Highly specific connections between the cortical lobe and thalamus. The 5 columns on the left show the cortical lobe and highly correlated thalamic regions in patients with IGE and healthy controls. In the winner-take-all map, each thalamic voxel is labeled according to the cortical lobe with the highest $t$ value. The $z$ plane coordinates indicate the Montreal Neurological Institute space.

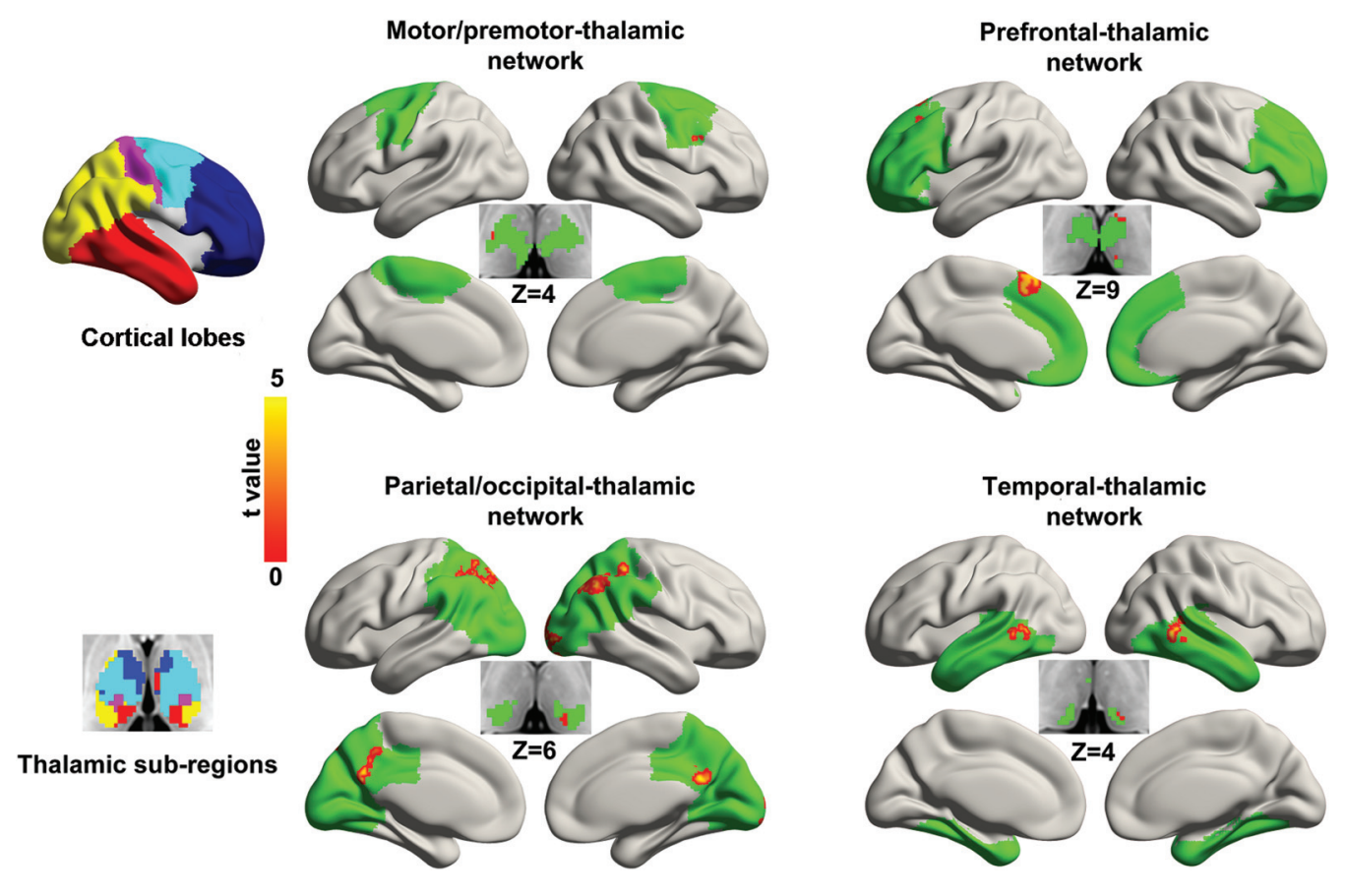

FIG 3. Comparison of voxelwise functional connectivity between groups. The left column shows cortical lobes and corresponding thalamic subregions obtained by using the winner-take-all approach. The right column shows the between-group difference in FCS. Abnormal voxels are labeled by warm colors and rendered on cortical lobe and thalamic subregions (green). The color scale represents $t$ values by using 2 -sample $t$ tests $(P<.05$, corrected). The $z$ plane coordinates indicate the Montreal Neurological Institute space.

\section{RESULTS}

\section{Identification of Functional Corticothalamic Networks}

In control subjects, each cortical lobe was connected to distinct, largely nonoverlapping parts of the thalamus (Fig 2), consistent with previous findings. ${ }^{13}$ Specifically, the prefrontal lobe showed maximal correlation with the mediodorsal and anterior nuclear areas; the motor/premotor cortex corresponded most strongly to the ventral anterior, lateral dorsal, and part of the medial nucleus; the somatosensory cortex corresponded to the ventral posterior nucleus; and the parietal/occipital and temporal cortices corresponded to the lateral and medial part of the pulvinar. Similar patterns were observed in patients. However, qualitative differences were observed between the groups. Compared with controls, the thalamic subregions connected to the prefrontal and motor/premotor cortices were expanded and shrunken, respectively, in patients. The changes may simply relate to the different sample sizes of each group, rather than pathologic alteration.

\section{Differences in Functional Connectivity Strength}

A between-group comparison indicated that FCS was increased in patients (Fig 3 and Table 2). In the prefrontal-thalamic network, the regions of increase were located in the left middle frontal gyrus, left supplementary motor area (SMA), left pulvinar, and left ventral anterior nucleus. The motor/premotor-thalamic network showed increased FCS in the right premotor cortex and right ventrolateral nucleus. The parietal/occipital-thalamic network showed increased FCS in the bilateral inferior parietal lobule, left precuneus, right middle occipital gyrus, and left pulvinar. The FCS in the sensory-thalamic network did not differ between groups. The temporal-thalamic network showed increased FCS in the bilateral middle temporal gyrus and left pulvinar.

No significant correlation was found between the FCS in any abnormal region and disease duration, onset age, or seizure frequency, even when the age at time of the scan was controlled by regression. 
Table 2: Regions in corticothalamic networks with abnormal FCS in patients with IGE

\begin{tabular}{|c|c|c|c|c|}
\hline Brain Regions & MNI $(X, Y, Z)$ & $\begin{array}{c}\text { Brodmann } \\
\text { Area }\end{array}$ & $\begin{array}{c}\text { Volume } \\
\left(\mathrm{mm}^{3}\right)\end{array}$ & $\begin{array}{c}\text { Peak } \\
t \text { Value }\end{array}$ \\
\hline \multicolumn{5}{|l|}{ Prefrontal-thalamic network } \\
\hline SMA/middle frontal gyrus (L) & $-30,20,60$ & $8 / 6$ & 2136 & 4.25 \\
\hline Pulvinar (L) & $-8,-24,8$ & - & 48 & 3.54 \\
\hline Ventral anterior nucleus (L) & $-8,-4,10$ & - & 24 & 3.47 \\
\hline \multicolumn{5}{|l|}{ Motor/premotor-thalamic network } \\
\hline Premotor cortex (R) & $46,14,34$ & 6 & 184 & 4.26 \\
\hline Ventrolateral nucleus (R) & $20,-14,10$ & - & 48 & 3.76 \\
\hline \multicolumn{5}{|l|}{ Parieto/occipital-thalamic network } \\
\hline Middle occipital gyrus (R) & $18,-102,-10$ & 18 & 880 & 4.38 \\
\hline Precuneus (R) & $6,-52,24$ & 31 & 888 & 4.88 \\
\hline Inferior parietal lobule (R) & $42,-66,46$ & $40 / 7$ & 2712 & 4.87 \\
\hline Inferior parietal lobule (L) & $-36,-62,50$ & $40 / 7$ & 2424 & 4.95 \\
\hline Pulvinar (L) & $-14,-28,6$ & - & 48 & 4.08 \\
\hline \multicolumn{5}{|l|}{ Temporal-thalamic network } \\
\hline Middle temporal gyrus (R) & $62,-48,4$ & $22 / 21$ & 560 & 4.88 \\
\hline Middle temporal gyrus (L) & $-66,-50,-2$ & 21 & 432 & 4.76 \\
\hline Pulvinar (L) & $-8,-24,2$ & - & 48 & 3.41 \\
\hline
\end{tabular}

Note:-L, left hemisphere; MNI, Montreal Neurological Institute; R, right hemisphere; -, not applicable.
The present findings also identified the SMA in a cohort of patients with IGE. In an fMRI study using an executive frontal lobe paradigm, patients with juvenile myoclonic epilepsy showed increased functional connectivity between the motor system and the frontoparietal cognitive networks, providing an explanation for how cognitive effects can cause myoclonic jerks in juvenile myoclonic epilepsy. ${ }^{24}$

It was suggested that the SMA, as a connector linking prefrontal cognitive areas and the motor system, may facilitate the occurrence of generalized seizures, a notion supported by alterations in anatomic connectivity of the SMA in juvenile myoclonic epilepsy. ${ }^{25}$ To estimate the corticothalamic connectivity in juvenile myoclonic epilepsy, we parcellated the thalamus into several nuclei

\section{DISCUSSION}

By measuring brain synchronization by using resting-state fMRI, we characterized corticothalamic networks in patients with IGE. We replicated the 5 corticothalamic networks described previously in both patients and controls, indicating highly organized patterns of coherent activity in the thalamus and cerebral cortex. Most important, voxelwise functional connectivity analysis revealed epicenters within corticothalamic networks in patients. The results suggest that components of the corticothalamic network are not uniformly involved in IGE but that specific epicenters may be crucial to understanding the pathophysiology of this disorder.

IGE is regarded as a network disorder ${ }^{1}$ involving abnormal corticothalamic connectivity. ${ }^{4}$ Wang et $\mathrm{al}^{8}$ identified abnormal thalamic nuclei in a group of patients with IGE by morphologic analysis, which provided the foundation for seed-based functional connectivity analysis. This revealed decreased connectivity between the medial dorsal nucleus and bilateral orbital frontal cortices, caudate nucleus, putamen, and amygdala. However, another study found a different pattern of connectivity by using a similar analytic strategy. ${ }^{21}$ This inconsistency is at least partly due to the different locations of seed regions in these studies. To avoid the bias of a predefined seed region in the present study, we performed a voxelwise functional connectivity analysis (expressed as FCS) between the thalamus and cortex. ${ }^{19}$ More important, the analysis was not restricted to any single corticothalamic network but considered each one independently. Brain regions with high FCS are network hubs that facilitate the integration and propagation of neural processing. ${ }^{19}$ Increased FCS in patients with IGE is consistent with a high excitatory state or neuronal synchronization $^{22}$ and suggests that certain brain regions may be responsible for rapidly spreading the generalized spike-waves to all areas.

The original corticothalamic theory of seizure generalization was largely based on evidence from animal studies on absence seizures. ${ }^{2-4}$ Recent neuroimaging studies have implicated prefrontal areas such as the SMA, medial prefrontal cortex, and orbital frontal cortex in the generation of generalized seizures. ${ }^{22-24}$ connected to different cortices by using probabilistic tractography. ${ }^{7}$ A decreased probability of a connection between the anterior thalamus and SMA was found in patients. A psychophysiologic interaction analysis of fMRI data found greater task-dependent functional connectivity between these anterior nuclei and the superior frontal cortex. Accordingly, we observed increased FCS in the anterior nuclei. Similar altered prefrontal-thalamic connectivity in different IGE subtypes suggests that these network changes may be a common feature of IGE. The identification of these key regions - especially the SMA—provides putative epicenters that can be targeted by clinical treatment strategies. Research across disciplines on these epicenters can advance our understanding of the pathophysiology of IGE. Because specific changes in functional connectivity may respond best to particular clinical treatments, ${ }^{26}$ our identification of aberrant corticothalamic epicenters may help in the development of improved therapies with fewer side effects. ${ }^{4}$

Because most patients experience sustained muscle rigidity and rapid muscle contractions/relaxations during seizures, it is reasonable to expect abnormalities in the motor-/sensorythalamic system. The premotor cortex and specific nuclei (pulvinar and ventrolateral nucleus) showed increased FCS within the motor-thalamic but not the sensory-thalamic network. This is analogous to the disruption of functional integrity in the sensorimotor network of the neocortex ${ }^{27}$ and may reflect the higher excitability of the motor system of patients with IGE compared with controls. ${ }^{28}$ Thus, the motor system of these patients may be more susceptible to behavioral symptoms driven by the SMA during cognitive tasks. ${ }^{24}$ Brain regions showing increased FCS in the parietal cortex (precuneus and inferior parietal lobule) belong to the posterior part of the default mode network. ${ }^{29}$ Structural and functional abnormalities have been reported by diffusion-weighted imaging, ${ }^{30}$ cortical thickness, ${ }^{6}$ and resting-state fMRI studies. ${ }^{27}$ The posterior default mode network and related subcortical structures have been associated with the loss of consciousness during seizures. ${ }^{31}$ The increased default mode network-pulvinar FCS 
in the current study is in accord with findings that default mode network activity increases several seconds before seizure onset. $^{32}$ Given that the default mode network-pulvinar network is associated with the default state of the human brain, ${ }^{31}$ increased synchrony with thalamic nuclei in the interictal or preictal state may facilitate seizures. ${ }^{33}$ In the temporal-thalamic network, the morphometric features of the posterior part of the middle temporal gyrus showed stronger correlation with those of the thalamus in patients with IGE than in controls. ${ }^{6}$

Our results provide additional evidence for abnormalities in this network at the functional level. Because the posterior part of the middle temporal gyrus has been suggested as a semantic hub,$^{34}$ our findings help explain abnormal semantic processing observed in many patients with IGE. In addition to corticothalamic involvement in the pathophysiology of IGE, increasing evidence underscores the importance of the striatal system. ${ }^{7,8,30}$ The striatum exerts an inhibitory effect on motor systems, ${ }^{35}$ and its interaction with the motor and premotor cortices enables the smooth execution of voluntary movements. ${ }^{36}$ Decreased control over the highly excitable network may also underlie the behavioral symptoms of seizures such as myoclonic jerks.

There were several limitations to this study. First, simultaneous electroencephalograms were not acquired to exclude fMRI data with GSWDs; thus, we did not know whether the abnormalities in corticothalamic networks were caused by epileptic discharges. In addition, the mono-/polytherapy undertaken by some patients may have confounded the results. Furthermore, epileptic activity is dynamic, and it is therefore difficult to determine its origin by using a static method. Finally, cognitive data and the socioeconomic status of patients and controls were not taken into account; however, it is unlikely that these factors contributed to the between-group differences because most of the participants were from middleclass families.

\section{CONCLUSIONS}

Key regions within corticothalamic networks were identified with abnormal FCS in patients with IGE through a resting-state functional connectivity analysis. The identification of these epicenters (the SMA, premotor cortex, pulvinar, ventrolateral nucleus, and default mode network-pulvinar network) provides support for the corticothalamic network theory of IGE pathophysiology.

Disclosures: Gong-Jun Ji, Zhiqiang Zhang, Qing Jiao, Yu-Feng Zang, Wei LiaoRELATED: Grants: National Natural Science Foundation of China.

\section{REFERENCES}

1. Berg AT, Berkovic SF, Brodie MJ, et al. Revised terminology and concepts for organization of seizures and epilepsies: report of the ILAE Commission on Classification and Terminology, 2005-2009. Epilepsia 2010;51:676-85

2. Meeren H, van Luijtelaar G, Lopes da Silva F, et al. Evolving concepts on the pathophysiology of absence seizures: the cortical focus theory. Arch Neurol 2005;62:371-76

3. Avoli M. A brief history on the oscillating roles of thalamus and cortex in absence seizures. Epilepsia 2012;53:779-89

4. Blumenfeld H. From molecules to networks: cortical/subcortical interactions in the pathophysiology of idiopathic generalized epilepsy. Epilepsia 2003;44(suppl 2):7-15
5. Gotman J, Grova C, Bagshaw A, et al. Generalized epileptic discharges show thalamocortical activation and suspension of the default state of the brain. Proc Natl Acad Sci U S A 2005;102:15236-40

6. Bernhardt BC, Rozen DA, Worsley KJ, et al. Thalamo-cortical network pathology in idiopathic generalized epilepsy: insights from MRI-based morphometric correlation analysis. Neuroimage 2009; 46:373-81

7. O’Muircheartaigh J, Vollmar C, Barker GJ, et al. Abnormal thalamocortical structural and functional connectivity in juvenile myoclonic epilepsy. Brain 2012;135:3635-44

8. Wang Z, Zhang Z, Jiao Q, et al. Impairments of thalamic nuclei in idiopathic generalized epilepsy revealed by a study combining morphological and functional connectivity MRI. PLoS One 2012;7:e39701

9. Szaflarski JP, DiFrancesco M, Hirschauer T, et al. Cortical and subcortical contributions to absence seizure onset examined with EEG/ fMRI. Epilepsy Behav 2010;18:404-13

10. Morel A, Magnin M, Jeanmonod D. Multiarchitectonic and stereotactic atlas of the human thalamus. J Comp Neurol 1997;387:588-630

11. Metzger CD, van der Werf YD, Walter M. Functional mapping of thalamic nuclei and their integration into cortico-striatal-thalamocortical loops via ultra-high resolution imaging: from animal anatomy to in vivo imaging in humans. Front Neurosci $2013 ; 7: 24$

12. Tyvaert L, Chassagnon S, Sadikot A, et al. Thalamic nuclei activity in idiopathic generalized epilepsy: an EEG-fMRI study. Neurology 2009;73:2018-22

13. Zhang D, Snyder AZ, Fox MD, et al. Intrinsic functional relations between human cerebral cortex and thalamus. $J$ Neurophysiol 2008;100:1740-48

14. Woodward ND, Karbasforoushan H, Heckers S. Thalamocortical dysconnectivity in schizophrenia. Am J Psychiatry 2012;169:1092-99

15. Anticevic A, Cole MW, Repovs G, et al. Characterizing thalamocortical disturbances in schizophrenia and bipolar illness. Cereb Cortex 2014;24:3116-30

16. Nair A, Treiber JM, Shukla DK, et al. Impaired thalamocortical connectivity in autism spectrum disorder: a study of functional and anatomical connectivity. Brain 2013;136:1942-55

17. Power JD, Barnes KA, Snyder AZ, et al. Spurious but systematic correlations in functional connectivity MRI networks arise from subject motion. Neuroimage 2012;59:2142-54

18. Fair DA, Bathula D, Mills KL, et al. Maturing thalamocortical functional connectivity across development. Front Syst Neurosci 2010;4:10

19. Tomasi D, Volkow ND. Functional connectivity density mapping. Proc Natl Acad Sci U S A 2010;107:9885-90

20. Zhang Z, Liao W, Chen H, et al. Altered functional-structural coupling of large-scale brain networks in idiopathic generalized epilepsy. Brain 2011;134:2912-28

21. Kim JB, Suh SI, Seo WK, et al. Altered thalamocortical functional connectivity in idiopathic generalized epilepsy. Epilepsia 2014;55:592-600

22. Bai X, Guo J, Killory B, et al. Resting functional connectivity between the hemispheres in childhood absence epilepsy. Neurology 2011;76:1960-67

23. Holmes MD, Brown M, Tucker DM. Are “generalized” seizures truly generalized? Evidence of localized mesial frontal and frontopolar discharges in absence. Epilepsia 2004;45:1568-79

24. Vollmar C, O’Muircheartaigh J, Barker GJ, et al. Motor system hyperconnectivity in juvenile myoclonic epilepsy: a cognitive functional magnetic resonance imaging study. Brain 2011;134:1710-19

25. Vollmar C, O’Muircheartaigh J, Symms MR, et al. Altered microstructural connectivity in juvenile myoclonic epilepsy: the missing link. Neurology 2012;78:1555-59

26. Fox MD, Buckner RL, Liu H, et al. Resting-state networks link invasive and noninvasive brain stimulation across diverse psychiatric and neurological diseases. Proc Natl Acad Sci U S A 2014;111:E4367-75

27. Wang Z, Lu G, Zhang Z, et al. Altered resting state networks in 
epileptic patients with generalized tonic-clonic seizures. Brain Res 2011;1374:134-41

28. Reutens DC, Berkovic SF, Macdonell RA, et al. Magnetic stimulation of the brain in generalized epilepsy: reversal of cortical hyperexcitability by anticonvulsants. Ann Neurol 1993;34:351-55

29. Raichle ME, MacLeod AM, Snyder AZ, et al. A default mode of brain function. Proc Natl Acad Sci U S A 2001;98:676-82

30. Yang T, Guo Z, Luo C, et al. White matter impairment in the basal ganglia-thalamocortical circuit of drug-naive childhood absence epilepsy. Epilepsy Res 2012;99:267-73

31. Blumenfeld H. Impaired consciousness in epilepsy. Lancet Neurol 2012;11:814-26

32. Benuzzi F, Mirandola L, Pugnaghi M, et al. Increased cortical BOLD signal anticipates generalized spike and wave discharges in adoles- cents and adults with idiopathic generalized epilepsies. Epilepsia 2012;53:622-30

33. Vaudano AE, Laufs H, Kiebel SJ, et al. Causal hierarchy within the thalamo-cortical network in spike and wave discharges. PLoS One 2009;4:e6475

34. Binder JR, Desai RH, Graves WW, et al. Where is the semantic system? A critical review and meta-analysis of 120 functional neuroimaging studies. Cereb Cortex 2009;19:2767-96

35. Stocco A, Lebiere C, Anderson JR. Conditional routing of information to the cortex: a model of the basal ganglia's role in cognitive coordination. Psychol Rev 2010;117:541-74

36. Nagano-Saito A, Martinu K, Monchi O. Function of basal ganglia in bridging cognitive and motor modules to perform an action. Front Neurosci 2014;8:187 